Japanese Psychological Research

1977, Vol. 19, No. 4, 166-173

\title{
INFORMED DISGRIMINATION REVERSALS AND STRATEGY LEARNING OF JAPANESE MONKEYS ${ }^{1}$
}

\author{
JUNJI KOMAKI ${ }^{2}$ \\ University of Kanazawa
}

\begin{abstract}
Two groups of three male Japanese monkeys were given one of two modified forms of successive reversal training (SRT) in WGTA. Prior to usual SRT trials of each reversal, three information trials were introduced on which either baited positive stimulus ( $\mathrm{P}$ stimulus group) or unbaited negative stimulus (N stimulus group) of new reversal was singly presented. Two-trial problems were given on three stages and LS problems on the last stage of the informed SRT's so as to assess the development of choice strategies. SRT group of the previous study was control group. N stimulus group was superior to SRT group in reversal performance and comparable to it in 2-trial and LS problems. P stimulus group, though superior to SRT group in reversal performance, was inferior to it in 2-trial and LS problems. It indicates that development of choice strategy needs something other than successful reversal performance. Implications of this and other findings were discussed on the assumption that level of attentiveness during training might be a determinant of strategy learning.
\end{abstract}

One of the remarkable influences of successive reversal training (SRT) is its effect on strategy performance of monkeys. In a previous study (Komaki, 1974) two groups of monkeys were given either SRT or overtraining of comparable trials. SRT group monkeys displayed, in the strategy probes of 2-trial problems, significantly superior "win-stay" and "loseshift" performance to the overtrained monkeys. Apparently SRT has the effect to improve the learning of these strategies. The present study is an attempt to reach an understanding of how SRT contributes to strategy learning by analyzing the influences of some SRT components on development of the strategies.

In the standard type of SRT the positive and negative values of paired stimuli are reversed every time each subject reaches

1 The present study was supported in part by Grant-in-Aid for Cooperative Study, Primate Research Institute of Kyoto University, 1975.

${ }^{2}$ Requests for reprints should be sent to Junji Komaki, Department of Psychology, Faculty of Law and Literature, University of Kanazawa, Maruno-uchi 1-1, Kanazawa 920, Japan. a criterion of learning. This aspect of SRT procedures introduces two important components which subjects must learn to follow; non-rewarding of a response to the new negative (previous positive) stimulus and rewarding of a response to the new positive (previous negative) stimulus. Because these components are essential to SRT, it is reasonable to assume that they act on the development of "winstay" and "lose-shift" strategies. The present study concentrated on these SRT components. And, in order to evaluate their contributions to the strategy learning, two modified forms of SRT to which either one of the components was additionally presented were devised: Two groups of monkeys were provided, prior to every stage of SRT, with either non-rewarding trials of the new negative stimulus ( $N$ stimulus trials) or rewarding trials of the new positive stimulus ( $P$ stimulus trials).

We are not in a position to propose any specific assumptions concerning the influences of the trials. But, as a guide for the present study, the following working hypothesis was proposed: $\mathrm{N}$ stimulus 
trial would force subjects to withhold response from a certain discriminative stimulus and thus have the effect of improving the learning of "lose-shift" strategy. On the other hand, $\mathrm{P}$ stimulus trial would enable subjects to make an approach response to a certain discriminative stimulus and enhance the development of "winstay" strategy. In other words, introduction of $\mathrm{N}$ and $\mathrm{P}$ stimulus trials would selectively reinforce "lose-shift" and "winstay" strategy respectively. On the basis of the temporary hypothesis, it was expected that the group provided with $\mathrm{N}$ stimulus trials ( $\mathrm{N}$ stimulus group) would manifest, in the strategy probes of 2-trial problems, better " lose-shift" performance and the group provided with $\mathbf{P}$ stimulus trials ( $\mathbf{P}$ stimulus group) would display better "win-stay" performance, when compared with a standard SRT group to which no such stimulus trials were introduced.

\section{METHOD}

\section{Subjects}

Six male adult Japanese monkeys (Macaca fuscata) were the subjects. They were wildborn monkeys and were reared more than two years and a half at the Primate Research Institute of Kyoto University. All the monkeys were experimentally naive. They were fed after their daily session in their individual cages where water was always available. Reward was either a raisin or a half peanut, depending on which the individual subject preferred.

Part of the data obtained in the previous study (Komaki, 1974) provided control scores. It was of the three male Japanese monkeys of SRT group which were given the standard SRT. They were comparable to the subjects of the present study in terms of age as well as past experience.

\section{Apparatus}

All the training and the problems were given to the subjects in a modified version of
WGTA (Komaki, 1974). Essentially, it was composed of two cages. A subject was introduced into one of the cages, and the stimulus was presented on a movable stimulus tray in the other cage. An opaque and a transparent screen separated the cages. The stimulus tray had three food wells on it, each one measuring $12 \mathrm{~cm}$ from edge to edge. The 63 pairs of stimuli used in the previous study were the discriminanda. The stimuli were three dimensional wooden objects, differentially colored, and varied in form and size. Three pairs were applied to the informed SRT and the remaining 60 pairs to 2-trial and 5-trial problems.

\section{Preliminary Training}

According to the schedule described by Murofushi (1966-67), subjects were given progressive stages of training. The criterion at the final stage of training was that each subject could displace an object covering one of the food wells as soon as the transparent screen was raised. The subjects took 151 to 209 trials (8 to 9 days), and were assigned to one of the two groups below in a counterbalanced order so as to produce two equated groups in terms of trials.

\section{Procedure}

General training procedure and design. A trial started at the opening of the opaque screen. A few seconds later, when the subject looked forward, the experimenter raised the transparent screen about $10 \mathrm{~cm}$ above the floor. As soon as the subject was rewarded for a correct response or displaced the incorrect stimulus, the screen was lowered. Then the opaque screen was lowered and the trial was terminated. Inter-trial interval was 25 to $30 \mathrm{sec}$ except in 2-trial problems.

All the subjects were given an original discrimination, three blocks of 20 informed reversals, six series of 2-trial problems, and twenty 5-trial LS problems. Some of the 2-trial problem series were interpolated within the informed SRT and the other series were presented after the SRT: One of the first three 2-trial problem serics was presented 
after each of the three reversal blocks and the remaining series were provided in succession after the third series of 2-trial problems.

Informed SRT. All the subjects were initially trained on an original discimination. One of the three stimulus pairs was assigned to one subject of each group, and the nonpreferred member of the pair was the correct stimulus. When each subject reached the criterion of ten consecutive correct responses, the informed SRT which would be extended over three blocks of 20 reversals was initiated. Every reversal of the informed SRT was composed of two phases; three information trials and usual reversal training. The information trial phase was differentiated between the two groups.

To the subjects of $\mathrm{P}$ stimulus group the negative stimulus of the previous discrimination (new positive stimulus) was presented singly over the center food well on the stimulus tray, and the response to it was rewarded. To the subjects of $\mathrm{N}$ stimulus group the previous positive stimulus (new negative stimulus) was presented singly, and the response to it was not rewarded. When the subject did not displace the unbaited stimulus within $30 \mathrm{sec}$, the trial was terminated and the response latency of 30 sec was recorded.

The reversal training phase was common to the two groups. Paired stimuli were presented over the two extreme food wells, and alternated between left and right according to a predetermined order. Twentyfour sequences were selected from the table by Fellows (1967) and combined to form six series of 48 trials. These series were rotated every six days. The criterion of reversal learning was ten consecutive correct responses. In the first block of the informed SRT, daily session was 48 trials. In the second and third SRT blocks, however, daily trials were 45 to 51. When the subject reached the reversal criterion within the last three trials of the daily 48 trials, any information trial of the new reversal was not given on the day. The information trials were given before the presentation of the 48 trials allotted to the next day.

Two-trial problems. All the monkeys re- ceived strategy behavior probes made of six series of 2-trial problems. Each of the subjects received one of the three series on the day next to the day when it finished each informed SRT block, and the remaining three series in succession after the third series of 2-trial problems.

Ten baited and ten unbaited 2-trial problems constituted each series and made up a daily session of 40 trials. The baited problem was to test "win-stay" strategy behavior. On the first trials of the baited problems both the paired stimuli were baited, and the stimulus chosen on the trial was the correct stimulus for the next trial. The unbaited problem was to test "lose-shift" behavior. On the first trials of the unbaited problems both the stimuli were not baited, and the stimulus not chosen was the correct stimulus for the next trial.

Positions of the paired stimuli were alternated on the second trials in half of the baited and the unbaited problems (differential problems), but not alternated in the remaining halves of the problems (duplicate problems). Thus, every 2-trial problem series consisted of four kinds of five problems involving four possible combinations : Baited-differential, baitedduplicate, unbaited-differential and unbaitedduplicate problems. These problems were arranged in sequence according to a predetermined order.

Sixty pairs of stimuli were also put in order to produce three sets of 20 pairs. These sets were given to three subjects in each group as the first three problem series to from a $3 \times 3$ Latin square. These sets were used again in the same sequence as the second three problem series. The interval between the problems was 35 to $40 \mathrm{sec}$ and that between trials $30 \mathrm{sec}$.

Five-trial problems. Following the 2-trial problems, the subjects undertook twenty 5trial problems for two days. These problems were usual object discrimination learning set problems with five intra-problem trials. Every daily session was 50 trials of ten problems which were composed of five baited and five unbaited problems. In the baited 5-trial problem both stimuli were baited on the first trial, and 
the object chosen was the correct stimulus for the subsequent four trials. Neither stimulus was baited on the initial trial of unbaited problem, and the object not chosen was the correct stimulus on the succeeding four trials.

These two sorts of problems and the position of the stimuli were arranged randomly. The set of 20 stimulus pairs which was used for the first and the fourth 2-trial problem series for each subject was adopted as the discriminanda for the 5-trial problems.

\section{Results}

\section{Information Trial Performance}

The measure of performance on the information trials was response speed, which was defined as a reciprocal of the time in seconds from the opening of the transparent screen to the displacement of the unbaited ( $N$ stimulus group) or the baited (P stimulus group) stimulus. During the SRT, speed increased in $P$ stimulus group and decreased in N stimulus group. $\mathbf{P}$ stimulus group monkeys attained their asymptotes ( 1.70 to 2.70 ) on the first half of the second SRT block. $\mathrm{N}$ stimulus group monkeys took more training and reached their asymptotic levels $(.20$ to .60$)$ in the last SRT block.

\section{Errors in SRT}

Both $\mathrm{N}$ and $\mathrm{P}$ stimulus trials reduced errors on early trials of the reversals. Evidence comes from the two separate analyses of error performance by $\mathbf{N}$ and $\mathbf{P}$ stimulus groups and the SRT group of the previous study (Komaki, 1974).

The first was the analysis of initial consecutive errors. These errors were obtained for each subject in each SRT block, and an analysis of variance of Groups $\times$ SRT Blocks was applied. SRT Blocks were repeated measures. The main effect of Groups was highly significant, $F(2,6)=$ $60.37, p<.01$. Mean consecutive errors per reversal was .53 in $\mathrm{N}$ stimulus group, 1.42 in $P$ stimulus group, and 2.02 in SRT group. Newman-Keuls tests showed that all the group differences were significant, $p$ 's $<.01$. It is evident, therefore, that $\mathrm{N}$ and $\mathrm{P}$ stimulus trials reduce early errors in reversal, and that $\mathrm{N}$ stimulus trials are more effective than $\mathrm{P}$ stimulus trials. These errors reached asymptotic levels in the second SRT block. The main effect of SRT Blocks was also significant, $F(2,12)=17.83, p<.01$. And, NewmanKeuls tests revealed significant reduction of the errors from the first to the second SRT block, $p<.01$, but did not from the second to the last SRT block. The interaction of Groups $\times$ SRT Blocks was not significant.

The second evidence was error performance by the three groups on the initial ten trials of the reversals. Errors on each of these trials were pooled for each SRT block, and an analysis of variance of Groups $\times$ SRT Blocks $\times$ Trials was applied. SRT Blocks and Trials were repeated measures. Groups effect was not significant. But, the main effects of SRT Blocks, $F(2,12)=25.58, p<.01$, and Trials, $F(9,54)=38.29, p<.01$, and the interaction of Groups $\times$ Trials, $F(18,54)=9.59$, $p<.01$, were significant. Errors on these trials, taken as a whole, significantly decreased in the second SRT block, $p<.01$, but there was no more reduction of the errors from the second to the third SRT block. Errors tended to decrease on later trials. However, as the significant interaction of Groups $\times$ Trials would imply, rates of the error reduction were different among the groups. Simple main effects (Winer, 1971, p. 545) of Trials were signifcant at $\mathrm{P}$ stimulus group, $F(9,54)=13.32$, $p<.01$, and SRT group, $F(9,54)=42.01$, $p<.01$, but not at $\mathrm{N}$ stimulus group. In addition, simple main effects of Groups were significant only at Trial $1, F(2,24)=$ $45.2, p<.01$, and Trial 2, $F(2,24)=6.1$, $p<.01$. Pairwise comparisons (Winer, 1971 , p. 544) showed that all the differences among the groups were significant on Trial 1, $p$ 's $<.01$, and that only the difference between $\mathrm{N}$ and SRT groups 
TABLE 1

Trials to achieve each SRT block of 20 reversals by each subject of the three groups (information trials not included)

\begin{tabular}{c|c|ccc}
\hline \multirow{2}{*}{ Groups } & Subjects & \multicolumn{3}{|c}{ SRT block } \\
\cline { 2 - 5 } N stimulus group & 1 & 728 & 335 & 336 \\
& 2 & 761 & 403 & 337 \\
& 3 & 795 & 419 & 466 \\
\hline \multirow{3}{*}{ P stimulus group } & 4 & 529 & 329 & 317 \\
& 5 & 678 & 369 & 400 \\
& 6 & 793 & 410 & 561 \\
SRT group (1974) & a & 538 & 420 & 426 \\
& b & 715 & 415 & 374 \\
& c & 704 & 476 & 376
\end{tabular}

was significant on Trial 2, $p<.01$. Error percentages on these trials, when all SRT blocks were pooled, are shown in Fig. 1 for the three groups.

\section{Reversal Trials}

Although $\mathrm{N}$ and $\mathrm{P}$ stimulus trials

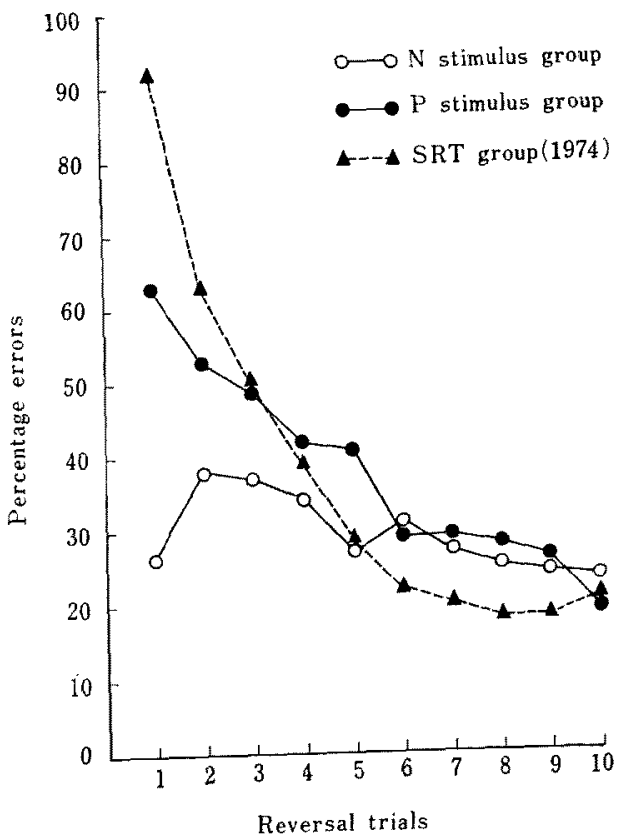

Fic. 1. Mean percentage of errors on each of the initial ten reversal trials (all reversals pooled). reduced early errors in reversals, their effects were not so great as to reduce training trials of reversals. Trials needed to achieve every SRT block (Table 1) were put to an analysis of variance of Groups $\times$ SRT Blocks of a repeated measures design. The main effect of SRT Blocks was highly significant, $F(2,12)=$ $99.92, p<.01$. However, neither the main effect of Groups nor the interaction of Groups $\times$ SRT Blocks was significant. Occurrence of errorless reversal was rare. Mean errorless reversal was 1.7 in P stimulus group and 4.0 in $\mathrm{N}$ stimulus group. These scores correspond to only 1.39 and $3.33 \%$ of all the reversals, respectively. Reversals achieved with one error or less were obtained for the three groups. Mean of these reversals was 5.3 in $\mathrm{P}$ stimulus group, 12.7 in $\mathrm{N}$ stimulus group and 2.0 in SRT group. An analysis of variance of Groups $\times$ SRT Blocks was applied. Only the main effect of SRT Blocks was significant, $F(2,12)=5.78, p<.05$.

\section{Two-trial Problem Performance}

Performance by the three groups in the six series of 2-trial problems is shown in Fig. 2. As seen in the figure, $\mathrm{N}$ stimulus group and SRT group displayed comparable performance, but apparently $P$ stimulus group was worse in strategy performance than the two groups.

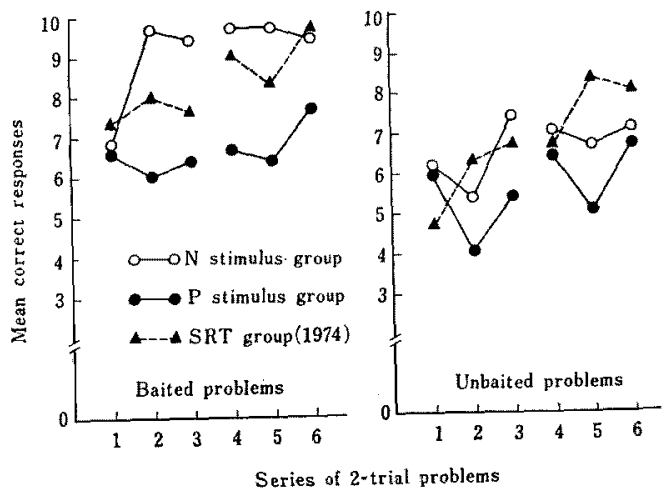

FIc. 2. Mean correct responses of the three groups in each series of 2-trial problems. 
Each 2-trial problem series was composed of 20 problems, five for each of four kinds. Percentages of correct response for each kind of problem were obtained for each series and were transformed into their inverse sine roots. And, an analysis of variance of Groups $\times$ Blocks (first three series and second three series of the problems) $\times$ Orders (serial position of the series within the block) $\times$ Tasks (baited and unbaited) $\times$ Cues (duplicate and differential) was applied to the transformed scores. The Blocks, Orders, Tasks and Cues were repeated measures.

The main effect of Groups was significant, $F(2,6)=5.90, p<.05$. NewmanKeuls tests revealed that $P$ stimulus group was significantly inferior to $N$ stimulus and SRT groups, $p$ 's $<.05$, and that there was no reliable difference between the latter groups. It is evident, therefore, that only the $\mathrm{N}$ stimulus informed SRT produced the strategy learning comparable to the standard SRT.

The main effects of Blocks, $F(1,6)=$ $8.14, p<.05$, and Cues, $F(1,6)=13.68$, $p<.025$, were significant, but the other main effects and all the interactions were not significant. All the subjects displayed better performance in the second block of the problem series. And their performance was better in duplicate problems than differential problems.

\section{Five-trial Problem Performance}

The superiority of $\mathrm{N}$ stimulus and SRT groups to $\mathrm{P}$ stimulus group was also observed in the 5-trial problems (Fig. 3). Percentage correct responses on each trial of baited and unbaited 5-trial problems were transformed into their inverse sine roots. Additionally, an analysis of variance of Groups $\times$ Tasks $\times$ Trials of a repeated measures design was applied.

The main effect of Groups was significant, $F(2,6)=17.71, p<.01$. NewmanKeuls tests indicated that $P$ stimulus group was significantly inferior to $\mathrm{N}$ stimulus group as well as SRT group, $p<.01$, and that the latter groups did not differ from each other. The trials effect was also significant, $F(3,18)=10.28, p<.01$. Pairwise comparisons by Newman-Keuls method revealed that the effect was mainly due to the significantly higher level of performance on the fifth trial than the second, $p<.01$, and than the third trial, $p<.05$, of the problems. The main effect of Tasks was not significant, nor were any of the interactions.

\section{Discussion}

Introduction of $\mathrm{N}$ and $\mathrm{P}$ stimulus trials proved to be helpful to reversal performance. $\mathrm{N}$ and $\mathrm{P}$ stimulus groups to which either sort of information trial was given in a block of three trials made less errors than SRT group on early trials of reversals. $\mathrm{N}$ stimulus group manifested better performance than $P$ stimulus group on the early trials. It indicates that $\mathrm{N}$ stimulus trials were more influential in guiding reversal choices. P stimulus trials, though helpful to reversal choices, were less effective than usual two-stimulus reversal trials. As seen in Fig. 1, the error percentage of $P$ stimulus group on the first reversal trials averaged about $63 \%$, considerably higher than that of SRT group on the fourth reversal trials. $\mathrm{N}$ stimulus trials seems to be superior or at least comparable to reversal training trials in guiding early

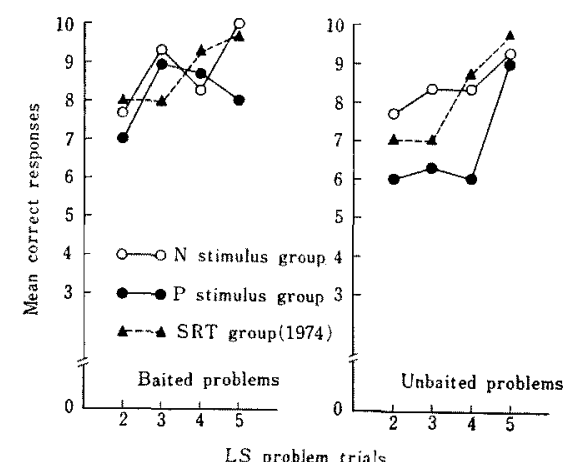

Fig. 3. Mean correct responses of the three groups on each trial of 5-trial problems. 
reversal choices. N stimulus group's error percentage on the first reversal trials was lower than SRT group's on the fourth reversal trials.

$\mathrm{N}$ as well as $\mathrm{P}$ stimulus trials were always given to the subjects just prior to the beginning of reversal phase. One might expect, therefore, that the presentation of these trials could inform the subjects of the coming of reversal and thus serve as "a sign for reversal." But, there was no indication that these trials actually acted as such a sign. Errorless reversals by the informed SRT groups were at best 3.33\% of all the reversals and far from the level displayed in the study by Riopelle and Copelan (1954).

$\mathrm{N}$ and $\mathrm{P}$ stimulus trials had differential influences on strategy learning itself. N stimulus group was comparable to SRT group in the strategy probes of 2-trial problems. On the other hand, $P$ stimulus group was inferior to SRT group not only in "lose-shift" performance but also in "win-stay" performance. These results were not expected. Concerning the influence of $\mathrm{P}$ stimulus trials, the working hypothesis was at first that these trials would improve the development of "win-stay" strategy. But, actually they impaired it. In addition, the influence of $P$ stimulus trials was broader than expected. The hypothesis predicted that these trials would affect only the "win-stay" strategy learning. But they were also deteriorative to the "lose-shift" learning.

$\mathrm{N}$ stimulus trials, unlike $\mathrm{P}$ stimulus trials, did not disturb the development of strategy learning. However, their influence on it was also different than expected. The working hypothesis was roughly that $N$ stimulus group would be better than SRT group in "lose-shift" behavior. This was not the case. On the contrary, $\mathrm{N}$ stimulus group happened to be better than SRT group in "win-stay" performance. As seen in Fig. 2, $\mathrm{N}$ stimulus group reached its asymptotic level of "winstay" performance on the second series of the problems. However, the apparent superiority of this group to SRT group was not statistically significant, perhaps due to ceiling effect. In light of these results, we have to alter the original conception that $\mathrm{N}$ and $\mathrm{P}$ stimulus trials can selectively reinforce "lose-shift" and "win-stay" strategies.

To explain these unexpected influences of the information trials, we have to pay attention at first to the dissociation between reversal performance and strategy learning which was found in $\mathrm{P}$ stimulus group. Through stimulus trials, this group achieved better reversal performance than SRT group. Nevertheless, the latter group was superior in strategy learning. This indicates that development of strategy learning needs something other than successful reversal performance.

We have not enough information to decide what is needed for strategy development. However, one of the previous findings (Komaki, 1974) provides some clue to an understanding of a condition for strategy learning. It is the strategy behavior of overtrained monkeys. These monkeys were given 1624 to 1638 extended trials of their original discrimination problems. They rarely made errors and manifested nearly complete series of correct responses during the overtraining. But, that series of correct responses was not effective for strategy learning. Their "win-stay" and " lose-shift" performance was inferior to SRT group monkeys. This is another instance of the dissociation between discriminative performance and strategy learning, because the overtrained monkeys, though conducting nearly perfect discriminative responses, scarcely learned strategies. While readily choosing correct stimulus, monkeys during overtraining seem to have been less attentive to discriminanda than they were in original training. This observation is suggestive. We can interpret this as implying that for monkeys to learn strategy in overtraining they must be as much attentive to dis- 
criminanda as in original discrimination training. This principle seems to be adaptable to explain the failure of strategy learning in $\mathbf{P}$ stimulus group and other findings.

In standard SRT monkeys are presented with a single pair of discriminanda the reward values of which are reversed each time they reach a criterion of learning. Thus, SRT group monkeys are forced to be attentive to the discriminanda and to maintain initial task level of attentiveness throughout the training to learn strategy. $\mathrm{P}$ stimulus group monkeys, on the other hand, do not keep original task level of attentiveness by the interpolation of $\mathrm{P}$ stimulus trials. In reversal phases of the informed SRT, they are forced to be as much attentive to the discriminanda as SRT group monkeys. But, on P stimulus trials their level of attention may be brought back to some lower level, because they are presented with only a baited stimulus and need not to do any discrimination to obtain reward. This interruption of high level attentiveness might be a cause for the lack of strategy learning in this group. On the contrary, $\mathrm{N}$ stimulus group must be alert even on their information trials. They have to learn not to make response to the presented stimulus. And, in doing this, they must presumably pay more attention to it than $\mathrm{P}$ stimulus group monkeys. As described above, there was a great difference between $\mathrm{N}$ and $\mathrm{P}$ stimulus groups in the degree of reward value learning of the presented stimulus. Between the first reversal trial errors of the two groups there was a difference of almost $40 \%$ (Fig. 1). Part of the difference may be a reflection of the different level of the attentiveness of the groups on the information trials.

Since the notable finding by Schusterman (1964) there has been an accumulation of evidence confirming the develop- ment of "win-stay" and "lose-shift" strategies during SRT. However, we have not known any definite way in which SRT can contribute to strategy learning. Implied in most SRT studies is a belief that execution of reversal performance or reversal choice response pattern which is achieved according to the repeated reversals of cue values will lead to the learning of those strategies. But, reversal choice performance is by no means sufficient for the learning. Strategy development needs something other than successful reversal performance. As described above, we have supposed that attentiveness level to discriminative stimulus is a determinant of strategy learning. But, this supposition is mostly derived of inferences and is not supported by any direct evidence. Further studies examining this and other determinants must be made to elucidate the conditions for strategy development.

\section{REFERENCES}

Fellows, B. J. 1967 Chance stimulus sequences for discrimination tasks. Psychological Bulletin, 67, 87-92.

KomakI, J, 1974 The influence of overtraining and successive reversal training on strategic behavior of Japanese monkeys. Japanese Psychological Research, 16, 149-156.

Murofushi, K. 1966-67 Interspecific contrasts in temperament: A comparison of three species of macaque monkeys. Delta Primates Research Center.

Riopelle, A.J., \& Copelan, E. L. 1954 Discrimination reversal to a sign. Fournal of Experimental Psychology, 48, 143-145.

Sahusterman, R.J. 1964 Successive discrimination-reversal training and multiple discrimination training in one-trial learning by chimpanzees. Fournal of Comparative and Physiological Psychology, 58, 153-156.

Winer, B. J. 1971 Statistical principles in experimental design. 2nd ed. New York: McGrawHill.

(Received Sept. 27, 1977) 